\title{
NATIVE SPECIES INDICATED FOR DEGRADED AREA RECOVERY IN WESTERN PARANÁ, BRAZIL ${ }^{1}$
}

\author{
Darlene Gris², Lívia Godinho Temponi ${ }^{3}$ e Thaís Regina Marcon²
}

\begin{abstract}
Colonization in the State of Paraná has culminated in the devastation of large forest areas in the entire State. Degraded area recovery programs have emphasized the utilization of native species, but often the species indicated for local reforestation areas are unknown, as those areas are little known floristically. This study aimed to survey native species indicated for reforestation of areas in the Western region of the State of Paraná, classify those species as pioneer, secondary, or climactic, and indicate places of occurrence of matrices where seeds of those species could be collected. Bibliographic surveys in the specialized literature and research in the Herbarium Museu Botânico Municipal de Curitiba (MBM) and Herbarium of Universidade Estadual do Oeste do Paraná (UNOP) were conducted to identify potential species for degraded area recovery in the study of Western region of Paraná. In all, 115 species were selected, of which 22 are pioneer, 73 are secondary, and 20 are climactic. The bibliographic surveys suggests that pioneer species are the most indicated for the initial processes in the degraded areas recovery, while secondary and climactic species play a major role in area enrichment.
\end{abstract}

Keywords: Reforestation, Pioneer species, Secondary species and climactic species.

\section{ESPÉCIES NATIVAS INDICADAS PARA RECUPERAÇÃO DE ÁREAS DEGRADADAS NO OESTE DO PARANÁ}

\begin{abstract}
RESUMO - A colonização no Estado do Paraná culminou na devastação de grandes áreas florestadas em todo o Estado. Os programas de restauração de áreas degradadas enfatizam a utilização de espécies nativas, mas, muitas vezes, não se conhecem as espécies indicadas para reflorestamentos locais, uma vez que essas áreas são pouco conhecidas floristicamente. Este trabalho teve como objetivo levantar as espécies nativas, classificando as como pioneiras, secundárias e climácicas, indicadas para o reflorestamento de áreas na região Oeste do Paraná, bem como apontar localidades de ocorrência das matrizes para a coleta de sementes dessas espécies. Foram realizadas pesquisas bibliográficas em literatura especializada e nos herbários Museu Botânico Municipal de Curitiba (MBM) e Herbário da Universidade Estadual, no Oeste do Paraná (UNOP), com o intuito de identificar as espécies potenciais para a recuperação de áreas degradadas na região Oeste do Paraná. Foram selecionadas 115 espécies, das quais 22 são pioneiras, 73 são secundárias e 20 são climácicas. A Revisão Bibliográfica sugere que as espécies pioneiras são as mais indicadas para os processos iniciais na recuperação de áreas degradadas, enquanto as espécies secundárias e clímax têm papel principal no enriquecimento da área.
\end{abstract}

Palavras-chave: Reflorestamento, Espécies pioneiras, Espécies secundárias e espécies climácicas.

\footnotetext{
${ }^{1}$ Recebido em 16.09.2010 e aceito para publicação em 15.11.2011.

${ }^{2}$ Programa de Pós-Graduação em Conservação e Manejo de Recursos Naturais, Universidade Estadual do Oeste do Paraná, UNIOESTE, Brasil.E-mail: <darlenegris@hotmail.com>e <tisy@hotmail.com>.

${ }^{3}$ Universidade Estadual do Oeste do Paraná, UNIOESTE, Brasil.. E-mail: <liviatemponi@yahoo.com.br>.
} 


\section{INTRODUCTION}

The Atlantic Rainforest comprises three floristic complexes: Dense Ombrophilous Forests, Seasonal Semidecidual or Decidual Forests, and Subtropical Ombrophilous Forests (VELOSO et al., 1991). Although those complexes have a number of elements and aspects in common, they are very diversified with regard to their physiognomies and compositions. The study area, located in western Paraná state, comprises both Seasonal Semidecidual Forests and Subtropical Ombrophilous Forests.

Seasonal Semidecidual Forests are located in temperate climate zones, at elevations between 400 and $800 \mathrm{~m}$ for the most part. The climate is humid, with moderate temperatures, hot summers, and annual rainfall ranging from 1000 to $1600 \mathrm{~mm}$. They comprise both fertile soils such as oxisols (terras roxas) and poor and sandy soils. Even in regions not far apart from each other, a highly diversified physiognomy is present; however, the literature lacks more detailed information on the floristic composition of that forest before it was severely devastated (CÂMARA, 1991).

The elevation limit of the Subtropical Ombrophilous or Araucaria Forests is about 500m, although single patches can be found in lower areas as well as in higher elevations up to $1800 \mathrm{~m}$. The climate is invariably humid, with moderate summers. Originally the Subtropical Ombrophilous Forest covered a vast area in southern Brazil. Today there are approximately $1600 \mathrm{~km}^{2}$ about $0.8 \%$ of this phytoecological unit, due to a progressive anthropic action. This vegetation is confined to a few remnants of its original condition; however, it still shows a high degree of conservation and high biodiversity (RÖGLIN et al., 2007).

Dramatic changes to the landscape and to the structure and functionality of ecosystems were necessary for the development of mankind on the planet. Changes caused by man sought to increase the productivity of elements of interest for mankind and their domestic animals, with no concern for the rest of the environment (REIS et al., 2006).

The development of degraded area recovery projects is indispensable in order to restore the balance between man and nature. This is only possible with the incorporation of rational and less impacting projects that will allow ecodevelopment (JACOMEL; MARANHO, 2005).

Revista Árvore, Viçosa-MG, v.36, n.1, p.113-125, 2012
Legal Reserves Areas and Permanent Preservation Areas are the main targets for the degraded ecosystems restoration. The global and effective deficit is estimated in at least one million hectares in the State of Paraná and 40 million hectares in Brazil (CARPANEZZI; CARPANEZZI, 2006).

Originally, the territory of the State of Paraná was almost entirely covered with Atlantic Rainforest, which occupied $85 \%$ of the state; currently, however, only a small fraction of those forests remains unchanged. Intense deforestation, caused by the implementation of livestock and farming activities, has left only fragments of those forests in the state (MAACK, 2002).

Evaluations about the biodiversity, number, and geographic location of those forest fragments are the most important means employed to determine conservation strategies, to recover the vegetation, and to reestablish connections that will maintain the gene flow among species (KERSTEN, 2006).

At present, it is necessary to conciliate productive areas with conservation areas, thus causing a synergy between those dramatically fragmented landscapes. Therefore, in order to maintain the quality of life on earth, the degraded areas recovery, especially with respect to increasing the connectivity between fragments, has become a vital action (REIS et al., 2006). Although several degraded area recovery and degraded ecosystem restoration studies have been conducted, there has been a knowledge gap with regard to the exclusive use of native species and especially with regard to the conduction of such studies in the Western part of Paraná inasmuch as existing degraded ecosystem restoration projects and studies refer to other regions of Paraná and to other states. As a result, a knowledge of native species indicated for degraded area recovery in the Western part of the State of Paraná is indispensable and justified.

The objective of this study was to conduct a survey to determine native pioneer, secondary, and climactic species, as well as to present localities of occurrence of matrices of species indicated for degraded areas reforestation in the Western region of Paraná.

\section{MATERIALAND METHODS}

Bibliographic studies on all the specialized literature on the present floristic in the Western region of Paraná (IBAMA, 1999; LORENZI, 2002; CARVALHO, 2003; 
CARPANEZZI; CARPANEZZI, 2006; CARVALHO, 2006) were conducted. Later, studies on the main herbaria with samples from the region, Museu Botânico Municipal de Curitiba (MBM) and Herbarium da Universidade Estadual do Oeste do Paraná (UNOP) were conducted to identify the species with natural occurrence in the Western region of Paraná that are consequently suitable for degraded area recovery in the study area.

For each species listed, it was selected the place of occurrence closer to the Western region of the state, according to the bioclimatic regions of the Paraná (EMBRAPA, 1986).

The species were classified into successional groups after Carpanezzi and Carpanezzi (2006) and Silva et al. (2004). According to those authors, the species present the following successional groups: pioneer species require much light during their lifetime; their initial growth is very fast with a short lifetime (from four to 30 years); they are important in the formation of seed banks; secondary species, despite of being light demanding, they can tolerate shading in the beginning of their lifetime, with a moderate to rapid initial growth and a lifetime from 25 to 100 years; and climactic species, which tolerate strong shading for many years or remain in the understory during their entire lifetime, grow slowly, and have a moderate to long lifetime, forming plantlet banks.

\section{RESULTS}

A total of 115 native species were found in the Western region of Paraná (Table 1), of which about $19 \%$ were classified as pioneer, $64 \%$ as secondary, and $17 \%$ as climactic with respect to their ecological groups.

Table 1 - Native species in the Western region of Paraná selected for degraded area recovery (Nomenclature updated according to APGIII (2009).

Tabela 1 - Espécies nativas da região Oeste do Paraná, selecionadas para a recuperação de áreas degradadas (Nomenclatura atualizada de acordo com a APGIII (2009).

\begin{tabular}{|c|c|c|c|c|c|}
\hline Família & Nome científico & Nome comum & $\begin{array}{l}\text { Grupo } \\
\text { ecológico }\end{array}$ & $\begin{array}{l}\text { Local de coleta/Regiões } \\
\text { Bioclimáticas do PR }\end{array}$ & Herbário \\
\hline Anacardiaceae & $\begin{array}{l}\text { Lithrea brasiliensis } \\
\text { Marchand }\end{array}$ & bugreiro & secundária & Mangueirinha / 1 & MBM 2018 \\
\hline Anacardiaceae & Schinus molle L. & aroeira-salsa & secundária & Cascavel / 2 & UNOP 5428 \\
\hline Anacardiaceae & $\begin{array}{l}\text { Schinus terebinthifolius } \\
\text { Raddi }\end{array}$ & aroeira & pioneira & Cascavel / 2 & UNOP 5427 \\
\hline Annonaceae & Annona cacans Warm. & ariticum & secundária & Catanduvas / 3 & MBM 202524 \\
\hline Annonaceae & $\begin{array}{l}\text { Annona neosalicifolia } \\
\text { H. Rainer }\end{array}$ & ariticum & secundária & Foz do Iguaçu / 3 & MBM 67892 \\
\hline Annonaceae & $\begin{array}{l}\text { Annona rugulosa (Schltdl.) } \\
\text { H.Rainer }\end{array}$ & ariticum & pioneira & Foz do Iguaçu/3 & UNOP 6714 \\
\hline Apocynaceae & $\begin{array}{l}\text { Aspidosperma polyneuron } \\
\text { Müll.Arg. }\end{array}$ & peroba rosa & clímax & Palotina / 4 & МBM 179437 \\
\hline Apocynaceae & $\begin{array}{l}\text { Tabernaemontana } \\
\text { fuchsiaefolia A. DC. }\end{array}$ & leiteiro & clímax & Palotina / 4 & UNOP 5416 \\
\hline Apocynaceae & $\begin{array}{l}\text { Tabernaemontana } \\
\text { catharinensis A.DC. }\end{array}$ & leiteirinho & secundária & Campo Mourão / 2 e 4 & UNOP 5292 \\
\hline Aquifoliaceae & Ilex dumosa Reissek & voadeira & secundária & Medianeira / 3 & MBM 7467 \\
\hline Aquifoliaceae & $\begin{array}{l}\text { Ilex paraguariensis } \\
\text { A.St.-Hil. }\end{array}$ & erva-mate & climax & Cascavel / 2 & UNOP 5392 \\
\hline Aquifoliaceae & $\begin{array}{l}\text { Ilex theezans Mart. } \\
\text { ex Reissek }\end{array}$ & caúna & pioneira & Laranjeiras do Sul / 1 & МВM 47918 \\
\hline Araucariaceae & $\begin{array}{l}\text { Araucaria angustifolia } \\
\text { (Bertol.) Kuntze }\end{array}$ & $\begin{array}{l}\text { pinheiro-do- } \\
\text { paraná }\end{array}$ & secundária & Cascavel / 2 & UNOP 1362 \\
\hline Arecaceae & Euterpe edulis Mart. & palmito & climax & Cianorte / 4 & МВМ 9399 \\
\hline Arecaceae & $\begin{array}{l}\text { Syagrus romanzoffiana } \\
\text { (Cham.) Glassman. }\end{array}$ & jerivá & clímax & Santa Helena/ 3 & UNOP 6641 \\
\hline Bignoniaceae & $\begin{array}{l}\text { Jacaranda micrantha } \\
\text { Cham. }\end{array}$ & carobão & pioneira & Cascavel / 2 & UNOP 5397 \\
\hline Bignoniaceae & Jacaranda puberula Cham. & caroba & secundária & Foz do Iguaçu / 3 & MBM 66522 \\
\hline
\end{tabular}

Revista Árvore, Viçosa-MG, v.36, n.1, p.113-125, 2012 
Tabela 1 - Cont.

Table 1 - Cont.

\begin{tabular}{|c|c|c|c|c|c|}
\hline Família & Nome científico & $\begin{array}{l}\text { Nome comum } \\
\text { ecológico }\end{array}$ & Grupo & Local de coleta & Herbário \\
\hline$\overline{\text { Bignoniaceae }}$ & $\begin{array}{l}\text { Handroanthus heptaphyllus } \\
\text { Mattos }\end{array}$ & ipê-roxo & secundária & Cascavel / 2 & UNOP 4625 \\
\hline Boraginaceae & $\begin{array}{l}\text { Cordia trichotoma (Vell.) } \\
\text { Arráb. ex Steud. }\end{array}$ & louro-pardo & secundária & Palotina / 4 & UNOP 5420 \\
\hline Boraginaceae & $\begin{array}{l}\text { Cordia americana (L.) } \\
\text { Gottschiling \& J.S. Mill. }\end{array}$ & guajuvira & secundária & Céu Azul / 3 & UNOP 5532 \\
\hline Canellaceae & $\begin{array}{l}\text { Cinnamodendron dinisii } \\
\text { Schwacke }\end{array}$ & pimenteira & secundária & Guarapuava / 1 & MBM 11259 \\
\hline Cannabaceae & $\begin{array}{l}\text { Trema micrantha } \\
\text { (L.) Blume }\end{array}$ & crindiúva & pioneira & Cascavel / 2 & MBM 17410 \\
\hline Caricaceae & $\begin{array}{l}\text { Jacaratia spinosa ( Aubl.) } \\
\text { A.DC. }\end{array}$ & jaracatiá & secundária & Foz do Iguaçu / 3 & UNOP 5637 \\
\hline Celastraceae & Maytenus robusta Reissek & garapoca & secundária & Foz do Iguaçu / 3 & MBM 2052 \\
\hline Celastraceae & $\begin{array}{l}\text { Maytenus ilicifolia } \\
\text { Mart. ex. Reissek }\end{array}$ & espinheira-santa & secundária & Cascavel / 2 & UNOP 4552 \\
\hline Euphorbiaceae & $\begin{array}{l}\text { Actinostemon concolor } \\
\text { (Spreng.) Müll.Arg. }\end{array}$ & $\begin{array}{l}\text { laranjeira-do- } \\
\text { mato }\end{array}$ & secundária & Foz do Iguaçu / 3 & UNOP 5560 \\
\hline Euphorbiaceae & $\begin{array}{l}\text { Alchornea glandulosa subsp. } \\
\text { iricurana (Casar) Secco }\end{array}$ & tapiá-graúdo & secundária & Dois Vizinhos / 1, 2 e 3 & UNOP 5194 \\
\hline Euphorbiaceae & $\begin{array}{l}\text { Alchornea sidifolia } \\
\text { Müll.Arg. }\end{array}$ & tapiá & secundária & $\begin{array}{l}\text { Nova Prata do } \\
\text { Iguaçu / } 3\end{array}$ & MBM 218803 \\
\hline Euphorbiaceae & Croton urucurana Baill. & sangra-da-água & pioneira & $\begin{array}{l}\text { Capitão Leonidas } \\
\text { Marques / } 3\end{array}$ & MBM 301786 \\
\hline Euphorbiaceae & $\begin{array}{l}\text { Sapium glandulosum } \\
\text { (L.) Morong. }\end{array}$ & pau de leite & secundária & Foz do Iguaçu / 3 & MBM 66503 \\
\hline Euphorbiaceae & $\begin{array}{l}\text { Sebastiania commersoniana } \\
\text { (Baill.) L.B.Sm. \& Downs }\end{array}$ & branquilho & secundária & Céu Azul / 3 & UNOP 5602 \\
\hline Euphorbiaceae & $\begin{array}{l}\text { Sebastiania schottiana } \\
\text { Müll.Arg. }\end{array}$ & branquilho & pioneira & Guaira / 6 & MBM 63628 \\
\hline Fabaceae & $\begin{array}{l}\text { Albizia hassleri (Chodat) } \\
\text { Burkart }\end{array}$ & farinha-seca & pioneira & Foz do Iguaçu / 3 & МВM 198687 \\
\hline Fabaceae & $\begin{array}{l}\text { Apuleia leiocarpa (Vogel) } \\
\text { J.F.Macbr. }\end{array}$ & grápia & secundária & Cruzeiro do Iguaçu / 3 & MBM 242507 \\
\hline Fabaceae & Calliandra brevipes Benth. & esponjinha & pioneira & Palotina / 4 & UNOP 5419 \\
\hline Fabaceae & Calliandra foliolosa Benth. & sarandi & secundária & Céu Azul / 3 & UNOP 5609 \\
\hline Fabaceae & Copaifera langsdorffii Desf. & $\begin{array}{l}\text { óleo-pardo } \\
\text { copaíba }\end{array}$ & climax & Foz do Iguaçu / 3 & MBM 67680 \\
\hline Fabaceae & $\begin{array}{l}\text { Dalbergia frutescens (Vell.) } \\
\text { Britton }\end{array}$ & rabo-de-bugio & pioneira & $\begin{array}{l}\text { Capitão Leonidas } \\
\text { Marques / } 3\end{array}$ & MBM 309162 \\
\hline Fabaceae & $\begin{array}{l}\text { Enterolobium contortisiliquum } \\
\text { (Vell.) Morong }\end{array}$ & timbaúva & secundária & Foz do Iguaçu /3 & UNOP 5414 \\
\hline Fabaceae & Holocalyx balansae Micheli & alecrim & climax & Cascavel / 2 & UNOP 4870 \\
\hline Fabaceae & Inga laurina (Sw.) Willd. & ingá, ingá branco & secundária & Guaíra / 6 & MBM 66595 \\
\hline Fabaceae & Inga marginata Willd. & ingá, ingá feijão & secundária & Foz do Iguaçu / 3 & MBM 234522 \\
\hline Fabaceae & $\begin{array}{l}\text { Inga vera subsp. affinis } \\
\text { (DC.)T.D.Penn. }\end{array}$ & ingá, ingá & secundária & $\begin{array}{l}\text { Capitão Leonidas } \\
\text { Marques / } 3\end{array}$ & MBM 304521 \\
\hline Fabaceae & Inga virescens Benth. & ingá & secundária & Foz do Iguaçu / 3 & МВM 66596 \\
\hline Fabaceae & $\begin{array}{l}\text { Lonchocarpus campestris } \\
\text { Mart. ex Benth. }\end{array}$ & rabo-de-bugio & secundária & Foz do Iguaçu/ 3 & UNOP 6713 \\
\hline Fabaceae & $\begin{array}{l}\text { Lonchocarpus muehlbergianus } \\
\text { Hassl. }\end{array}$ & timbó-graúdo & secundária & Foz do Iguaçu / 3 & МBM 66643 \\
\hline Fabaceae & Machaerium brasiliense Vogel & jacarandá & secundária & Foz do Iguaçu/ 3 & UNOP 6725 \\
\hline Fabaceae & $\begin{array}{l}\text { Machaerium stipitatum } \\
\text { (DC.) Vogel }\end{array}$ & jacarandá & secundária & Guaira / 6 & MBM 42402 \\
\hline
\end{tabular}

Revista Árvore, Viçosa-MG, v.36, n.1, p.113-125, 2012 
Tabela 1 - Cont.

Table 1 - Cont

\begin{tabular}{|c|c|c|c|c|c|}
\hline Família & Nome científico & $\begin{array}{l}\text { Nome comum } \\
\text { ecológico }\end{array}$ & Grupo & Local de coleta & Herbário \\
\hline Fabaceae & $\begin{array}{l}\text { Mimosa bimucronata } \\
\text { (DC.) Kuntze }\end{array}$ & maricá & pioneira & $\begin{array}{l}\text { Capitão Leonidas } \\
\text { Marques / } 3\end{array}$ & MBM 206934 \\
\hline Fabaceae & $\begin{array}{l}\text { Myrocarpus frondosus } \\
\text { Allemão }\end{array}$ & cabreúva & secundária & Catanduvas / 2 & МВМ 93889 \\
\hline Fabaceae & $\begin{array}{l}\text { Parapiptadenia rigida } \\
\text { (Benth.) Brenan }\end{array}$ & angico-vermelho & secundária & Palotina / 4 & UNOP 5418 \\
\hline Fabaceae & $\begin{array}{l}\text { Peltophorum dubium } \\
\text { (Spreng.)Taub. }\end{array}$ & canafístula & secundária & Palotina / 4 & UNOP 5417 \\
\hline Fabaceae & Pterogyne nitens Tul. & amendoim-bravo & secundária & Santa Helena /3 & MBM 2956 \\
\hline Fabaceae & $\begin{array}{l}\text { Schizolobium parahyba } \\
\text { (Vell.) Blake }\end{array}$ & guapuruvu & secundária & Cascavel / 2 & UNOP 4843 \\
\hline Fabaceae & $\begin{array}{l}\text { Senegalia polyphylla (DC.) } \\
\text { Britton \& Rose }\end{array}$ & monjoleiro & secundária & Foz do Iguaçu/ 3 & UNOP 6722 \\
\hline Fabaceae & $\begin{array}{l}\text { Senna pendula (Humb.\& } \\
\text { Bonpl.ex Willd). H.S.Irwin } \\
\text { \& Barneby }\end{array}$ & fedegoso & pioneira & Cascavel / 2 & UNOP 5426 \\
\hline Lamiaceae & $\begin{array}{l}\text { Vitex megapotamica } \\
\text { (Spreng.) Moldenke }\end{array}$ & tarumã & secundária & Foz do Iguaçu / 3 & МBM 67566 \\
\hline Lauraceae & $\begin{array}{l}\text { Cryptocarya aschersoniana } \\
\text { Mez. }\end{array}$ & canela-pururuca & clímax & Palmital / 1 e 2 & MBM 154463 \\
\hline Lauraceae & $\begin{array}{l}\text { Endlicheria paniculata } \\
\text { (Spreng.) J.F.Macbr. }\end{array}$ & canela & clímax & Foz do Iguaçu /3 & UNOP 5549 \\
\hline Lauraceae & Nectandra grandiflora Nees & canela-fedida & clímax & Marmeleiro / 1 & MBM 5698 \\
\hline Lauraceae & Nectandra lanceolata Nees & canela branca & secundária & $\begin{array}{l}\text { Dois Vizinhos } \\
/ 1,2 \text { e } 3\end{array}$ & UNOP 5160 \\
\hline Lauraceae & $\begin{array}{l}\text { Nectandra megapotamica } \\
\text { (Spreng.) Mez }\end{array}$ & canela-amarela & climax & Foz do Iguaçu / 3 & MBM 234482 \\
\hline Lauraceae & $\begin{array}{l}\text { Ocotea diospyrifolia } \\
\text { (Meisn.) Mez }\end{array}$ & canela & pioneira & Foz do Iguaçu / 3 & MBM 132867 \\
\hline Lauraceae & Ocotea puberula (Rich.) Nees & canela-guaicá & secundária & Foz do Iguaçu / 3 & UNOP 5631 \\
\hline Lauraceae & $\begin{array}{l}\text { Ocotea pulchella } \\
\text { (Nees \& Mart.) Mez }\end{array}$ & canela-lageana & secundária & $\begin{array}{l}\text { Marechal Candido } \\
\text { Rondon / } 3\end{array}$ & MBM 66436 \\
\hline Lecythidaceae & $\begin{array}{l}\text { Cariniana legalis (Mart. ) } \\
\text { Kuntze }\end{array}$ & jequitibá rosa & secundária & Guaíra / 6 & МВМ 39274 \\
\hline Malvaceae & $\begin{array}{l}\text { Bastardiopsis densiflora } \\
\text { (Hook. \& Arn.) Hassl. }\end{array}$ & louro-branco & pioneira & Céu Azul / 3 & МВМ 308892 \\
\hline Malvaceae & $\begin{array}{l}\text { Ceiba speciosa (A.St.-Hil.) } \\
\text { Ravenna }\end{array}$ & paineira & secundária & Cascavel / 2 & UNOP 4634 \\
\hline Malvaceae & Heliocarpus americanus L. & jangada-brava & pioneira & Foz do Iguaçu/ 3 & UNOP 6717 \\
\hline Malvaceae & $\begin{array}{l}\text { Luehea divaricata Mart. } \\
\& \text { Zucc. }\end{array}$ & açoita-cavalo & secundária & Palotina / 4 & UNOP 5415 \\
\hline Meliaceae & $\begin{array}{l}\text { Cabralea canjerana } \\
\text { (Vell.) Mart. }\end{array}$ & canjerana & secundária & Foz do Iguaçu / 3 & UNOP 5422 \\
\hline Meliaceae & Cedrela fissilis Vell. & cedro & secundária & Palotina / 4 & UNOP 5430 \\
\hline Meliaceae & Guarea kunthiana A. Juss. & peloteira & secundária & Foz do Iguaçu / 3 & UNOP 5425 \\
\hline Meliaceae & Guarea macrophylla Vahl & baga-de-morcego & secundária & Foz do Iguaçu / 3 & UNOP 4520 \\
\hline Meliaceae & Trichilia casaretti C. DC. & catiguá & secundária & Foz do Iguaçu / 3 & UNOP 5654 \\
\hline Meliaceae & Trichilia catigua A.Juss. & catiguá & secundária & Céu Azul / 3 & МВМ 297147 \\
\hline Meliaceae & Trichilia elegans A.Juss. & $\begin{array}{l}\text { catiguá-de-folha- } \\
\text { miúda }\end{array}$ & secundária & Foz do Iguaçu / 3 & UNOP 4517 \\
\hline Meliaceae & Trichilia pallens C.DC. & catiguá & secundária & Três Barras / 3 & МВM 167455 \\
\hline Meliaceae & Trichilia pallida Sw. & catiguá & secundária & Foz do Iguaçu / 3 & МВM 144941 \\
\hline Monimiaceae & $\begin{array}{l}\text { Hennecartia omphalandra } \\
\text { J.Poiss. }\end{array}$ & canema & $\operatorname{climax}$ & Céu Azul / 3 & UNOP 5612 \\
\hline
\end{tabular}


Tabela 1 - Cont.

Table 1 - Cont

\begin{tabular}{|c|c|c|c|c|c|}
\hline Família & Nome científico & $\begin{array}{l}\text { Nome comum } \\
\text { ecológico }\end{array}$ & Grupo & Local de coleta & Herbário \\
\hline Monimiaceae & Mollinedia clavigera Tul. & pimenteira & secundária & Goio-Erê / 4 & MBM 308935 \\
\hline Moraceae & $\begin{array}{l}\text { Ficus adhatodifolia Schott } \\
\text { ex Spreng. }\end{array}$ & figueira & secundária & Céu Azul /4 & МВM 67991 \\
\hline Moraceae & Ficus insipida Willd. & figueira-do-brejo & secundária & Foz do Iguaçu/3 & UNOP 6724 \\
\hline Moraceae & $\begin{array}{l}\text { Sorocea bonplandii (Baill.) } \\
\text { W.C.Burger et al. }\end{array}$ & pau-cincho & secundária & Céu Azul / 3 & UNOP 5568 \\
\hline Myrsinaceae & Myrsine umbellata Mart. & capororocão & secundária & $\begin{array}{l}\text { Capitão Leonidas } \\
\text { Marques / } 3\end{array}$ & MBM 296652 \\
\hline Myrtaceae & $\begin{array}{l}\text { Blepharocalyx salicifolius } \\
\text { (Kunth) O.Berg }\end{array}$ & murta & clímax & Foz do Iguaçu/ 3 & UNOP 6727 \\
\hline Myrtaceae & $\begin{array}{l}\text { Calycorectes riedelianus } \\
\text { O. Berg. }\end{array}$ & cambuí & secundária & Guaíra / 6 & МВМ 76497 \\
\hline Myrtaceae & $\begin{array}{l}\text { Campomanesia xanthocarpa } \\
\text { (Mart.) O.Berg. }\end{array}$ & guabirova & climax & Cascavel / 2 & UNOP 4569 \\
\hline Myrtaceae & Eugenia florida DC. & cambuí & clímax & Foz do Iguaçu /3 & UNOP 6729 \\
\hline Myrtaceae & Eugenia uniflora L. & pitanga & climax & Cascavel / 2 & UNOP 4570 \\
\hline Myrtaceae & Myrcia splendens (Sw.) DC. & guamirim-branco & secundária & $\begin{array}{l}\text { Tuneiras do } \\
\text { Oeste / } 4\end{array}$ & MBM 294727 \\
\hline Phytolaccaceae & $\begin{array}{l}\text { Gallesia integrifolia } \\
\text { (Spreng.) Harms }\end{array}$ & pau-d'alho & secundária & $\begin{array}{l}\text { Francisco } \\
\text { Beltrão / } 1\end{array}$ & MBM 238617 \\
\hline Podocarpaceae & $\begin{array}{l}\text { Podocarpus lambertii } \\
\text { Klotzsch ex Endl. }\end{array}$ & pinheiro-bravo & secundária & Cascavel / 2 & UNOP 5396 \\
\hline Polygonaceae & Ruprechtia laxiflora Meisn. & $\begin{array}{l}\text { marmeleiro- } \\
\text { bravo }\end{array}$ & secundária & Foz do Iguaçu/3 & UNOP 6718 \\
\hline Proteaceae & $\begin{array}{l}\text { Roupala montana var. } \\
\text { brasiliensis (Klotzsch) } \\
\text { K.S.Edwards }\end{array}$ & carvalho- & clímax & Céu Azul / 3 & MBM 296895 \\
\hline Rhamnaceae & $\begin{array}{l}\text { Colubrina glandulosa } \\
\text { Perkins }\end{array}$ & sobrasil & secundária & $\begin{array}{l}\text { Campo Mourão } \\
\text { / } 2 \text { e } 4\end{array}$ & MBM 29811 \\
\hline Rosaceae & Prunus myrtifolia (L.) Urb. & pessegueiro-bravo & secundária & Cascavel / 2 & UNOP 4792 \\
\hline Rosaceae & $\begin{array}{l}\text { Prunus brasiliensis (Cham. } \\
\text { \& Schltdl.) D.Dietr. }\end{array}$ & $\begin{array}{l}\text { pessegueiro-do- } \\
\text { mato }\end{array}$ & secundária & Foz do Iguaçu / 3 & MBM 259620 \\
\hline Rutaceae & $\begin{array}{l}\text { Balfourodendron riedelianum } \\
\text { (Engl.) Engl. }\end{array}$ & pau-marfim & secundária & Foz do Iguaçu / 3 & UNOP 5547 \\
\hline Rutaceae & Hellietta apiculata Benth. & canela-de-veado & secundária & Foz do Iguaçu / 3 & MBM 66340 \\
\hline Rutaceae & $\begin{array}{l}\text { Zanthoxylum rhoifolium } \\
\text { Lam. }\end{array}$ & mamica-de-porca & pioneira & $\begin{array}{l}\text { Marechal Candido } \\
\text { Rondon / } 3\end{array}$ & MBM 66341 \\
\hline Salicaceae & Casearia decandra Jacq. & guaçatunga-miúda & secundária & Foz do Iguaçu / 3 & МВM 67198 \\
\hline Salicaceae & Casearia obliqua Spreng. & guaçatunga-graúda & secundária & Marmeleiro / 1 & MBM 3743 \\
\hline Salicaceae & Casearia sylvestris $\mathrm{Sw}$. & cafezeiro-bravo & climax & Cascavel / 2 & UNOP 4731 \\
\hline Sapindaceae & $\begin{array}{l}\text { Allophylus edulis (A.St. } \\
\text {-Hil.et al.) Hieron ex } \\
\text { Niederl. }\end{array}$ & vacum & pioneira & Foz do Iguaçu / 3 & UNOP 4518 \\
\hline Sapindaceae & $\begin{array}{l}\text { Allophylus guaraniticus } \\
\text { (A.St.-Hil.) Radlk. }\end{array}$ & vacum & pioneira & Foz do Iguaçu / 3 & MBM 173029 \\
\hline Sapindaceae & Cupania vernalis Cambess. & cuvatã & secundária & $\begin{array}{l}\text { Nova Prata do } \\
\text { Iguaçu / } 3\end{array}$ & MBM 217970 \\
\hline Sapindaceae & $\begin{array}{l}\text { Diatenopteryx sorbifolia } \\
\text { Radlk. }\end{array}$ & maria-preta & secundária & Foz do Iguaçu / 3 & UNOP 5583 \\
\hline Sapindaceae & Matayba elaeagnoides Radlk. & miguel-pintado & climax & $\begin{array}{l}\text { Dois Vizinhos } \\
/ 1,2 \text { e } 3\end{array}$ & UNOP 5169 \\
\hline Sapotaceae & $\begin{array}{l}\text { Chrysophyllum gonocarpum } \\
\text { (Mart. \& Eichler ex Miq.) } \\
\text { Engl. }\end{array}$ & aguaí & climax & Foz do Iguaçu / 3 & UNOP 5634 \\
\hline
\end{tabular}

Revista Árvore, Viçosa-MG, v.36, n.1, p.113-125, 2012 


\begin{tabular}{|c|c|c|c|c|c|}
\hline Família & Nome científico & $\begin{array}{l}\text { Nome comum } \\
\text { ecológico }\end{array}$ & Grupo & Local de coleta & Herbário \\
\hline Solanaceae & Solanum mauritianum Scop. & fumo-bravo & pioneira & Foz do Iguaçu / 3 & MBM 345423 \\
\hline Solanaceae & $\begin{array}{l}\text { Solanum sanctae-catharinae } \\
\text { Dunal }\end{array}$ & canema & pioneira & Foz do Iguaçu / 3 & МВM 182361 \\
\hline Symplocaceae & $\begin{array}{l}\text { Symplocos uniflora } \\
\text { (Pohl.) Benth. }\end{array}$ & maria-mole & secundária & $\begin{array}{l}\text { Foz do Iguaçu } \\
/ 3\end{array}$ & МBM 5100 \\
\hline Urticaceae & $\begin{array}{l}\text { Cecropia pachystachya } \\
\text { Trécul }\end{array}$ & embaúba branca & pioneira & Foz do Iguaçu / 3 & MBM 238742 \\
\hline Urticaceae & $\begin{array}{l}\text { Urera baccifera (L.) } \\
\text { Gaudich. ex Wedd. }\end{array}$ & urtigão & pioneira & Foz do Iguaçu / 3 & МBM 191567 \\
\hline
\end{tabular}

In the bioclimatic regions of Paraná (EMBRAPA FLORESTAS, 1986) five had been shown as areas of occurrence of native species of Seasonal Semidecidual Forests and Subtropical Ombrophilous Forests. The regions with greater number of species were areas 2 and 3, with 24 and 71 species, respectively. The 29 remaining species had been registered in three bioclimatic regions: 1 ; 4 e 6 (Table 1 ).

\section{DISCUSSION}

The recovery of ecosystems can be improved by means of plant species that facilitate natural succession and accelerate the establishment of other species in the area, especially in recently-exposed surfaces (CHADA et al., 2004; CAMPELLO and FARIA, 2004, CONNEL; SLATYER, 1977).

Kageyama et al. (1994); Kageyama and Gandara (2004) observed that a certain hierarchy exists for light requirements from pioneer to climactic species and that each group requires distinct light intensities.

Pioneer species are very important in the ecological succession process inasmuch as they are less demanding with regard to the soil and have shorter life cycles that require much light during the entire cycle. These are fast-growing species that provide soil protection and microclimate conditions that are required for the establishment of species that belong to later successional stages. These species facilitate natural succession and their fruits serve as an attractive to the integrated fauna, which brings seeds from other areas (BOTELHO et al., 1996; DAVIDE and FARIA, 1996; GALETTI; STOZT, 1996; REBRAF, 2006).
Out of the 22 pioneer species found, three were often used in reforestation projects in the Western region of Paraná: Calliandra brevipes Benth.; Schinus terebinthifolius Raddi and Croton urucurana Baill.

Calliandra brevipes is a species that occurs mainly from Paraná to Rio Grande do Sul. The species is indifferent to soil physical conditions and is tolerant to frosts (CARPANEZZI; CARPANEZZI, 2006). It is often associated with river banks in Subtropical Ombrophilous Forest and Seasonal Semidecidual Forest regions.

Another example is Schinus terebinthifolius (pink pepper or aroeira) which is an evergreen, heliophyte, and pioneer plant common on the banks of rivers, streams and in humid lowlands of secondary formations; however, it also grows in dry and poor soils (LORENZI, 2002). This species is recommended for the recovery of less fertile soils (such as shallow, rocky, hydromorphic, or saline soils) because of its ruggedness, aggressiveness, and pioneer character, and fast growth (CARVALHO, 1988). Carvalho (2003) stated that because of its zoochorous dispersal, pink pepper is one of the most sought-after species by the avifauna, which is the most important disseminator in our midst.

A third example is sangra d'água (Croton urucurana) a deciduous, heliophyte species that typically occurs in very humid, swampy areas (LORENZI, 2002). It is a very fast-growing plant, reaching about $4 \mathrm{~m}$ in height two years after planting (MAXIMANO, 2008). It is frequently used for riparian forest regeneration because of its ability to withstand long periods under water and the ability to resist to a water column depletion area of up to 1 meter and withstand seasonal frosts (ASSAD-LUDEWIGS, 1989; BARBOSA, 1989; FARIA

Revista Árvore, Viçosa-MG, v.36, n.1, p.113-125, 2012 
et al., 1996). This species is also helpful for faunistic recovery, offering shelter and food such as extrafloral nectar (VERA, 1994). Pollination is anemophilous in open environments and it is occasionally performed by bees (PIRES, 2004); dispersal occurs by explosive dehiscence, flowers are melliferous and leaves and fruits provide food for many species of fish (BRAGA; GOMIERO, 2003; MAXIMANO, 2008).

Secondary species play an important role in ecological succession because of their rapid growth and large or medium-sized canopies, providing shading for climax species; they are indicated for area enrichment (CARPANEZZI; CARPANEZZI, 2006).

Amid the 73 secondary species, three were largely distributed in the region of the study (Cabralea canjerana (Vell.) Mart.; Cedrela fissilis Vell. and Luehea divaricata Mart. \& Zucc.), they were frequently registered in the Parque Nacional do Iguaçu and cited in the literature as promising species for reforestation in degraded areas in the Western region of Paraná.

One example is canjarana (Cabralea canjerana), whose fruits are highly ecological valued because of their high nutritional content; the juicy arillus can be consumed by several species of birds and small mammals, thus disseminating the seeds. For this reason its addition is indispensable in the composition of heterogeneous reforestations in areas appointed for permanent preservation (APREMAVI, 2009; LORENZI, 2002). The species is also highly plastic, that is, it has great capacity of undergoing phenotypic variations (induced by the environment) as a form of adaptation without the occurrence of genotypic variation, and it becomes adapted to various levels of solar radiation, under different lighting conditions, in experiments conducted in nurseries (SOUSA-SILVA et al., 1999; FUZETO; LOMÔNACO, 2000; FELFILI et al., 2001).

Another example is cedar (Cedrela fissilis), a deciduous, heliophyte or sciophyte plant, typical of semideciduous forests. It occurs from Rio Grande do Sul to Minas Gerais, especially in decidual and Atlantic rainforests.

Its pollen, nectar, and fruit provide food for the fauna, resulting in seed dispersal and consequently in forest revegetation (VASCONCELOS; AGUIAR, 1982; SCHUCH, 2005). Because this species thrives in humid soils, it is recommended for the degraded ecosystems restoration and for the recovery of riparian forests in sites where flooding occurs (DURIGAN; NOGUEIRA, 1990). In addition to these characteristics, Paiva and Poggiani (2000) verified that Cedrela fissilis shows fast growth in diameter, providing shading quickly for climax species.

A third example is açoita-cavalo (Luehea divaricata), a deciduous, heliophyte species, typical of riparian forests and galleries, indicated for the recovery of semi-hydromorphic and non-hydromorphic areas, frequently found in secondary formations (CURCIO et al. 2007; LORENZI, 2002).

It is mainly found in Dense Ombrophilous Forests and in Subtropical Ombrophilous Forests, in Alluvial and Montane formations, in addition to Seasonal Semidecidual and Decidual Forests (KLEIN, 1984; SILVA and MARCONI, 1990; VACCARO et al., 1999; LONGHI, and BRENA, 1999; CARVALHO, 1996).

Açoita-cavalo (Luehea divaricata) is recommended for crops in Permanent Preservation Areas, on steep slopes, river banks and in areas where the soil is periodically flooded, since it can withstand short-term periodic floods and moderate waterlogging (TORRES et al., 1992). In addition, dormancy does not occur in this species, thus facilitating germination. It is a fast growing plant, which also tolerates shading when young as well as low winter temperatures, such as those that occur in the State of Paraná; however, it has low resistance to late frosts (CARVALHO, 2003). According to Anton et al. (2002), this species can be recommended for the degraded areas regeneration because it has a considerable number of individuals in cases of ecological succession.

Moreira (2004) mentioned that Luehea divaricata is a very promising species for the revegetation of areas because of its high survival index and fast growth, in addition to a significant canopy area, allowing the development of climactic species that do not tolerate substantial incidence of sunlight during germination.

Climax species become settled after pioneer and secondary species create favorable conditions for their establishment (REIS, 2006). They are more demanding species with respect to soil type and amount of light, with slow growth and life cycles from moderate to long (CARPANEZZI; CARPANEZZI, 2006); they should be included in area recovery processes as a means of providing area enrichment. 
Of the 20 climax species found, Campomanesia xanthocarpa (Mart.) O.Berg. and Tabernaemontana fuchsiaefolia A. DC. Miers, are representative in the Western of Paraná, having a large number of individuals in the studied area, being recommended for its enrichment due to attract the fauna.

One such example is Campomanesia xanthocarpa, or guabiroba, a deciduous, heliophyte, and selective hygrophyte species. It has been classified as a secondary initial (VACCARO et al., 1999; LONGHI and BRENA, 1999), late secondary (DIAS et al., 1998), or shadetolerant climactic species (LORENZI, 2002; PINTO, et al. 2005). Its area of occurrence comprises the states of Minas Gerais, São Paulo, and Mato Grosso do Sul down to Rio Grande do Sul; it is very abundant in the humid regions of montane forests, such as semideciduous forests and pine forests (BRITEZ et al., 1992), and is very common in the Latifoliate Semideciduous Forest of the Paraná River Basin (LORENZI, 2002). Due to its abundant fructification, its fruit is consumed by several bird species and constitutes the favorite food of some Psittacidae such as parrots, in addition to other wild animals (CARVALHO, 2003; NERI et al., 2005). It thrives on well-drained river banks, providing excellent food for fish (MAIXNER; FERREIRA, 1976). It is also one of the species in Subtropical Ombrophilous Forests with the highest deposition of litter and macronutrients, with great importance in the forest structure (BRITEZ et al., 1992).

Another example is Tabernaemontana fuchsiaefolia, known as leiteiro or forquilheira, an evergreen, heliophyte, pioneer plant typical of the semideciduous forest of the São Paulo Plateau (LORENZI, 2002). It occurs in Rio de Janeiro, São Paulo, and Paraná, especially in Seasonal Semideciduous Forests. It has been classified as a climactic species by Carpanezzi and Carpanezzi (2006); nevertheless, its presence in the initial stages of the ecological succession has been demonstrated, suggesting that it is a pioneer or an initial secondary plant. Its fruit is much appreciated by the avifauna, which eat the red arillus that involves the seeds, and it is important in the composition of heterogeneous reforestations planned for the degraded Permanent Preservation Areas recovery. Coan et al. (2002) and Rosa (2003) suggests that leiteiro is indicated for the revegetation of degraded areas because the species is native to the Atlantic Rainforest and is attractive to birds that promote its dispersal.
Currently, degraded area recovery programs use native tree species almost exclusively. However, little knowledge is available on the most indicated species for local reforestation, especially in areas that are little known floristically, such as Western Paraná. Among other factors, this leads to the use of exotic species, favoring local biological contamination and increasing degradation (REIS et al., 2003).

The sampled areas are characterized by the presence of Seasonal Semidecidual Forests and Subtropical Ombrophilous Forests and it includes transition areas between them. The confirmed occurrence of these native species in the areas allows their use in reforestation projects in the region, with no risk of biological contamination (ZILER, 2007).

Despite the great number of species presented here for the region, these data are mentioned only for the species present in the revised literature, as well as in studies on the above-mentioned herbaria. Considering the history of the agricultural exploration and the increasing demand of the listed species for the reforestation of the region, an initial list is necessary. However, the Western Paraná region requires floristic studies and new surveys in these areas, the real number of native species probably will be even greater than the value represented here.

\section{CONCLUSION}

Pioneer species stand out because of their ruggedness, aggressiveness, and pioneer character, which are extremely important in the initial stage of the recovery process, since their inclusion makes the environment more favorable for the development of secondary and climactic species. One example of pioneer plant is Schinus terebinthifolius. In addition to the characteristics mentioned above, its fruit is frequently consumed by the fauna, as well as the fruit of Croton urucurana. Another important characteristic that should be emphasized is the ability to withstand the intense cold that occurs during winter in that region. The pioneer species Croton urucurana and Calliandra brevipes, as well as the secondary species Luehea divaricata should be highlighted with regard to this trait.

Traits that favor zoochory are indicated as being important inasmuch as they help seed dispersal and facilitate the nucleation process, accelerating recovery of the area. The pollen, nectar, and fruit of the secondary 
species Cedrela fissilis and Cabralea canjerana can be mentioned as examples. This is also an essential trait in climactic species, important for area enrichment, such as Campomanesia xanthocarpa and Tabernaemontana fuchsiaefolia that have juicy arillae that serve to attract avifauna, mainly.

\section{ACKNOWLEDGEMENTS}

The authors thank CNPq for granting a scientific initiation scholarship to the third author, Centro de Ciências Biológicas e da Saúde, Universidade Estadual do Oeste do Paraná, for financial support and the herbarium curator MBM for lending the material.

\section{REFERENCE}

ANTON, C. S. et al. Regeneração de Luehea divaricata Mart. E outras espécies em área degradada na região de Selvíria - MS. In: SIMPÓSIO NACIONAL SOBRE RECUPERAÇÃO DE ÁREAS DEGRADADAS, 5., 2002, Belo Horizonte. Anais.. Belo Horizonte, 2002. v.1. p.1-536.

ANGIOSPERM PHYLOGENY GROUP - APG. An update of the Angiosperm Phylogeny Group classification for the orders and families of flowering plants: APG III. Botanical Journal of the Linnean Society, v.161, p.105-121, 2009.

APREMAVI Canjerana. A árvore da madeira vermelha. Disponível em: <http:// www.apremavi.org.br/noticias/apremavi/553/ canjerana-a-arvore-da-madeira-vermelha $>$. Acesso em: 11 de out. de 2009.

ASSAD-LUDEWIGS, I. Y. et al. Propagação, crescimento e aspectos ecofisiológicos em Croton urucurana Baill. (Euphorbiaceae), arbórea nativa pioneira de mata ciliar. In: SIMPÓSIO SOBRE MATA CILIAR, 1989, Campinas. Anais... Campinas: Fundação Cargill, 1989. p.284-298.

BARBOSA, J. M. et al. Estudos dos efeitos da periodicidade da inundação sobre o vigor das sementes e desenvolvimento de plântulas para oito espécies ocorrentes em mata ciiar. In: SIMPÓSIO SOBRE MATA CILIAR, 1989, Campinas. Anais... Campinas: Fundação Cargill, 1989. p.310-319.

Revista Árvore, Viçosa-MG, v.36, n.1, p.113-125, 2012
BOTELLHO, S. A.; DAVIDE, A. C.; FARIA, J. M. R. Desenvolvimento inicial de seis espécies florestais nativas em dois sítios, na região sul de minas gerais. Cerne, v.2, n.1, p.4-13, 1996.

BRAGA, F. M. S.; GOMIERO, L. M. O lambari Astyanax altiparanae (Characidae) pode ser um dispersor de sementes? Maringá: Acta Scientiarum. Biological Sciences, v.25, n.2, p.353-360, 2003.

BRITEZ, R. M. et al. Deposição estacional de serrapilheira e macronutrientes em uma floresta de araucária, São Mateus do Sul, Paraná. Revista do Instituto Florestal, v.4, n.3, p.766-772, 1992.

CÂMARA, I. G. Plano de Ação para a Mata Atlântica. São Paulo: Fundação SOS Mata Atlântica, 1991. 57p.

CARPANEZZI, A. A.; CARPANEZZI, O. T. B. Espécies nativas recomendadas para recuperação ambiental no Estado do Paraná, em solos não degradados. Colombo: Embrapa Florestas, 2006. 57p.

CARVALHO, P. E. R. Potencialidades e restrições da regeneração artificial de espécies madeireiras nativas do Paraná. In: CONGRESSO FLORESTAL DO PARANÁ, Curitiba, PR, Anais... Curitiba: 1988.

CARVALHO, P. E. R. Influência da intensidade luminosa e do substrato no crescimento, no conteúdo de clorofila e na fotossintese de Cabralea canjerana (Vell.) Mart. subsp. canjerana, Calophyllum brasiliense Camb. e Enterolobium robustum (Vell.) Mart. em Benth.,na fase juvenil. 1996. Tese (Doutorado em Engenharia Florestal) Universidade Federal do Paraná, Curitiba, 1996.

CARVALHO, P. E. R. Espécies arbóreas brasileiras. Brasília: Embrapa, 2003. v.1. 1039p.

CARVALHO, P. E. R. Espécies arbóreas brasileiras. Brasília: Embrapa, 2006. v.2. 627p.

CHADA, S. S.; CAMPELLO, E. F. C.; FARIA, S. M. Sucessão vegetal em uma encosta reflorestada com leguminosas arbóreas em Angra dos Reis, RJ. Revista Árvore, v.28, n.6, p.801-809, 2004. 
COAN, L. F. B. et al. Recuperação de área degradada por construção de rodovia na Praia Mole, Florianópolis, SC. In: CONGRESSO BRASILEIRO DE SISTEMAS AGROFLORESTAIS, 4., 2002, Ilhéus. Anais... Ilhéus: 2002.

CONNELL, J. H.; SLATYER, R. O. Mechanisms of succession in natural communities and their role in community stability and organization.

The Americam Naturalist, v.111, n.982, p.1119-1144, 1977.

CURCIO, G. R. et al. Recomendação de espécies arbóreas nativas, por tipo de solo, para recuperação ambiental das margens da represa do rio Irai, Pinhais, PR. Floresta, v.37, n.1, p.113-122, 2007.

DIAS, M. C. et al. Composição florística e fitossociologia do componente arbóreo das florestas ciliares do Rio Iapó, na Bacia do Rio Tibagi, Tibagi, PR. Revista Brasileira de Botânica, v.21, n.2, p.183-195, 1998.

DURIGAN, G.; NOGUEIRA, J. C.

Recomposição de Matas Ciliares. São Paulo: Instituto Florestal, 1990. 14p. (IF. Série Registros, 4).

\section{EMPRESA BRASILEIRA DE PESQUISA} AGROPECUÁRIA - EMBRAPA. Centro Nacional de Pesquisa de Florestas, Zoneamento ecológico para plantios florestais no Estado do Paraná. Curitiba: 1986. 89p.

FARIA, J. M. R. et al. Comportamento de espécies florestais de mata ciliar em área de depleção do reservatório da usina hidrelétrica de Camargos Itutinga, MG. Cerne, v.2, n.1, 1996.

FELFILI, J. M. et al. Recuperação da vegetação de Matas de Galeria: estudos de caso no Distrito Federal e Entorno. In: RIBEIRO, J. F.; FONSECA, C. E. L.; SOUZA SILVA, J. C. (Org.). Cerrado: caracterização e recuperação de Matas de Galeria. Planaltina: Embrapa/CPAC, 2001. p.815-867.

FUZETO, A. P.; LOMÔNACO, C. Potencial plástico de Cabralea canjerana subsp. Polytricha (Adr. Juss.) Penn. (Meliaceae) e seu papel na formação de ecótipos em áreas de cerrado e vereda, Uberlândia, MG. Revista Brasileira de Botânica, v.23, n.2, p.169-176, 2000.
GALETTI, M. E.; STOZT, D. Miconia hypoleuca (Melastomataceae) como espéciechave para aves frugívoras no sudeste do Brasil. Revista Brasileira de Biologia, v.56, n.2, p.435-439, 1996.

IBAMA. Plano de Manejo do Parque Nacional do Iguaçu. Brasília: Diário Oficial da União. 1999.

JACOMEL, M.; MARANHO, L. T. Avaliação de um modelo de recuperação de floresta ombrófila mista (fom) em áreas degradadas por atividades agropecuárias. Revista Unicenp de Biologia e Saúde, v.1, n.4, p.9-10, 2005.

KAGEYAMA, P. Y. et al. Revegetação de áreas degradadas: modelos de consorciação com alta diversidade. In: SIMPÓSIO NACIONAL SOBRE RECUPERAÇÃO DE ÁREAS DEGRADADAS, 2., 1994, Foz do Iguaçu. Anais... Curitiba: FUPEF, 1994. p.569-576.

KAGEYAMA, P.; GANDARA, F. B. Recuperação de áreas ciliares. In: RODRIGUES, R. R.; LEITÃO FILHO, H. F., (Ed.). Matas ciliares conservação e recuperação. São Paulo: Edusp/Fapesp, 2004. p.249-269.

KERSTEN, R. A. Epifitismo vascular na Bacia do Alto Iguaçu, Paraná. 2006. Tese (Doutorado em Engenharia Florestal) Universidade Federal do Paraná, Curitiba, 2006.

KLEIN, R. M. O aspecto dinâmico do pinheiro brasileiro. Sellowia, v.12, n.12, p.17-44, 1960.

KLEIN, R. M. Meliáceas. Itajaí: Herbário Barbosa Rodrigues, 1984. 138p.

LORENZI, H. Árvores Brasileiras: manual de identificação e cultivo de plantas arbóreas do Brasil. 2.ed. Nova Odessa: 2002. v.1. 384p.

MAACK, R. Geografia física do estado do Paraná. 3.ed. Curitiba: Imprensa Oficial, 2002.

MAIXNER, A. E.; FERREIRA, L. A. B. Contribuição ao estudo das essências florestais e frutíferas nativas no Estado do Rio Grande do Sul - II. Trigo e Soja, n.18, p.3-20, 1976. 
MAXimano, N. A. Avaliação do crescimento inicial de vinte e nove espécies florestais no entorno de uma nascente pontual em processo de recuperação. 2008. Monografia (Graduação em Gestão Ambiental) - Escola Agrotécnica Federal de Inconfidentes-MG, Inconfidentes, 2008.

MOREIRA, P. R. Manejo do solo e recomposição da vegetação com vistas a recuperação de áreas degradadas pela extração de bauxita, Poços de Caldas, MG. 2004. Tese (Doutorado em Ciências Biológicas) - Universidade Estadual Paulista, Rio Claro, 2004.

NERI, A. V. et al. Regeneração de espécies nativas lenhosas sob plantio de Eucalyptus em área de Cerrado na Floresta Nacional de Paraopeba, MG, Brasil. Acta Botanica Brasilica, v.19, n.2., p.369-376, 2005.

PAIVA, A. V.; POGGIANI, F. Crescimento de mudas de espécies arbóreas nativas plantadas no sub-bosque de um fragmento florestal. Scientia Forestalis, n.57, p.141-151, 2000.

PIRES, M. M. Y. et al. Biologia floral de Croton urucurana Baill. (Euphorbiaceae) ocorrente em vegetação ripária da ilha Porto Rico, Porto Rico, Estado do Paraná, Brasil. Acta Scientiarum. Biological Sciences Maringá, v.26, n.2, p.209-215, 2004.

PINTO, L. V. A. et al. Estudo da vegetação como subsídios para propostas de recuperação das nascentes da Bacia Hidrográfica do Ribeirão Santa Cruz, Lavras, MG. Revista Árvore, v.29, n.5, p.775-793, 2005.

\section{INSTITUTO REDE BRASILEIRA}

AGROFLORESTAL - REBRAF. Proteção e restauração da área do entorno do Parque Estadual dos Três Picos. Relatório de Atividades de Campo. 2006.

REIS, A. et al. Restauração de áreas degradadas: a nucleação como base para incrementar os processos sucessionais. Revista Natureza e Conservação, v.1, n.1, p.28-36, 2003.

Revista Árvore, Viçosa-MG, v.36, n.1, p.113-125, 2012
REIS, A.; TRES, D. R.; BECHARA, F. C. A nucleação como novo paradigma na restauração ecológica: "Espaço para o imprevisível” In: SIMPÓSIO SOBRE RECUPERAÇÃO DE ÁREAS DEGRADADAS COM ÊNFASE EM MATAS CILIARES E WORKSHOP SOBRE RECUPERAÇÃO DE ÁREAS DEGRADADAS NO ESTADO DE SÃO PAULO: AVALIAÇÃO DAAPLICAÇÃO E APRIMORAMENTO DA RESOLUÇÃO SMA 47/03, 2006, São Paulo. Anais... São Paulo: Instituto de Botânica de São Paulo, 2006.

RÖGLIN, A.; WEBER, K. S.; SANQUETTA, C. R. Estrutura Horizontal de um fragmento de Floresta Ombrófila Mista em São João do Triunfo-PR. In: CONGRESSO DE ECOLOGIA DO BRASIL, 8., 2007, Caxambu. Anais... Curitiba: UFPR, 2007. p.1-2.

ROSA, G. A. B. Dispersão de sementes por aves em uma área de reflorestamento misto em Botucatu, SP. 2003. Dissertação (Mestrado em Ecologia) - Universidade Estadual de Campinas, Campinas, 2003.

\section{SCHUCH, D. R. Recuperação de um trecho de mata ciliar do rio Caeté, município de Urussanga, Santa} Catarina. 2005. Monografia (Pós-graduação em Gestão de Recursos Naturais) - Universidade do Extremo Sul Catarinense, Criciúma, 2005.

SILVA, F. C.; MARCONI, L. P. Fitossociologia de uma floresta com araucária em Colombo, PR. Boletim de Pesquisa Florestal, n.20, p.23-38, 1990.

SILVA, C. T. et al. Avaliação temporal da florística arbórea de uma floresta secundária no município de Viçosa, Minas Gerais. Revista Árvore, v.28, n.3, p.429-441, 2004.

SOUSA-SILVA, J. C. et al. Repartição de biomassa de Cabralea canjerana sob diferentes condições de sombreamento. Boletim Ezechias Paulo Heringer, v.4, p.80-89, 1999.

TORRES, R. B. et al. Espécies florestais nativas para plantio em áreas de brejo. $\mathbf{O}$ Agronômico, v.44, p.6-13, 1992.

VACCARO, S.; LONGHI, S. J.; BRENA, D. A. Aspectos da composição florística e categorias sucessionais do estrato arbóreo de três subseres de uma floresta estacional decidual no município de santa Tereza - RS. Ciência Florestal, v.9, n.1, p.1-18, 1999. 
VASCONCELOS, L. E. M.; AGUIAR, O. T. A alimentação de Aloucatta fusca Geof. (Primates, Cebidae). Silvicultura em São Paulo, v.16A, p.1727-1730, 1982. (Edição dos anais do $1^{\circ}$ Congresso Nacional sobre Essências Nativas, 1982, Campos do Jordão)

VELOSO, P. H. P.; RANGEL FILHO, A. L. R.; LIMA, J. C. A. Classificação da vegetação brasileira, adaptada a um sistema universal. Rio de Janeiro: IBGE - Departamento de Recursos Naturais e Estudos Ambientais, 1991. 123p.
VERA, C. V. M. Visitação por formigas e produção de néctar nos nectários extraflorais de Croton urucurana Baill (Euphorbiaceae), ao longo de um ano. 1994. 53f. Monografia (Bacharelado em Ecologia) - Instituto de Biociências, Universidade Estadual Paulista. Rio Claro, 1994.

ZILLER, S. R.; ZALBA, S. Propostas de ação para prevenção e controle de espécies exóticas invasoras. Natureza e Conservação, v.5, n.2, p.8-15, 2007. 